\title{
Formaldehyde Biosensor with Formaldehyde Dehydrogenase Adsorped on Carbon Electrode Modified with Polypyrrole and Carbon Nanotube
}

\author{
Mingwei Wang, Shuhai Jiang, Yi Chen, Xiang Chen, Li Zhao, Juankun Zhang*, Jinfeng Xu \\ Key Laboratory of Industrial Microbiology, Ministry of Education, College of Biotechnology, \\ Tianjin Key Laboratory of Industry Microbiology, College of Biotechnology, \\ Tianjin University of Science\&Technology, Tianjin, 300457, China \\ Email: zhangjk@tust.edu.cn
}

Received 2012

\begin{abstract}
In this study, a carbon electrode electroless polymerization of polypyrrole, which formed a layer of conductive film interface,then absorped a layer of carbon nanotube particles by the way of self-assembled, is studied. The modified electrode is used to electrostatic adherence of formaldehyde dehydrogenase, with Nafion solution capped. Moreover this paper discusses the work of electrode position, $\mathrm{PH}$ value and scanning speed on the influence of the electrode response, the response potential is $-1.2 \mathrm{v}$, the optimal PH value is 7.5, and we also review the linear range of this electrode, we discovered taht this biosensor have a good linear relationship with the concentration of $1 \mathrm{ug} / \mathrm{ml}-360 \mathrm{ug} / \mathrm{ml}$, the Correlation coefficient is 0.9983 . In addition, with the stability of this electrode tested, it can be stored $15 \mathrm{~d}$ at $4^{\circ} \mathrm{C}$.
\end{abstract}

Keywords: Formaldehyde Dehydrogenase; Eletrostatic Self-assembly; Biosensor

\section{Introduction}

Formaldehyde has been identified as carcinogenic and teratogenic substances by the World Health Organization, and it is the recognized source of allergy and a potentially strong mutagens, ranking second on the list of priority control of toxic chemicals in china[1]. Formaldehyde on human health is mainly manifested in the abnormal sense of smell, irritation, allergy, pulmonary function abnormalities, abnormal liver function and immune function abnormalities[2].

Making use of the catalytic properties of formaldehyde dehydrogenase for detecting formaldehyde, is not only easy to operate and specific, but also suitable for everyday use in the workplace. For example, Vianello's group[3] have coupled formaldehyde dehydrogenase on field-effect transistor, to detect airborne formaldehyde content by ion FET sensitive transistive, and the detection limit is $0.1 \mathrm{ug} / \mathrm{ml}$, much lower than the critical value of the occupational exposure. Mitsubavashi's group[4] have fixed formaldehyde dehydrogenase on a platinum electrode, to detect the production of NADH when enzyme reactions happen, which can reflect the content of formaldehyde. In this study, the linear range is $40-300 \mathrm{ug} / \mathrm{ml}$. M. Ben Ali's group [13] manufactured a biosensor with formaldehyde dehydrogenase, which was recombinated by Genetic engineering, and used this biosensor to detect trace amounts of formaldehyde with stripping voltammetry. Kohji. Mitsubayashi [14] developed a convenient biochemical chip for testing wood materials in gaseous formaldehyde. Lilach Bareket [15] developed a electrochemical biosensor based on carbon nanotubes for detecting formaldehyde release from cancer precursors drugs. Yaroslav [16] fixed formaldehyde dehydrogenase and cofactors on the surface of electrode, which avoided adding coenzyme and glutathione when detecting substrates, and the range of this biosensor is $10 \mathrm{mM}-200 \mathrm{mM}$.

In this study, we used the technology of electrostatic selfassembly for sdsorbing enzyme. Firstly, we polymerized polypyrrole on the surface of carbon electrode, Then we made it adsorb carbon nanotubes.At last, we put it in the solution containing formalde dehydrogenase, for adsorbing enzyme by electrostatic self-assembly. By this way, we produced a new and excellent biosensor for detecting formaldehyde.

\section{Experiment}

\subsection{Reagents}

Formaldehyde dehydrogenase was produced according to the papers of $[9,10]$. Pyrrole is purchased from the company of ke wei of tian jin.Carbon nanotubes was obtained in Shenzhen Nanotech Port Co,Ltd. Potassium ferricyanide is purchased from the company of Chemical Reagent of Yong Da in TianJin. All other reagents like as Na2HPO4.12H2O, NaH2PO4.H2O, $\mathrm{NaCl}$, Anhydrous alcohol were of analytical grade.Pyrrole was freshly distilled before use.Double distilled water was used for the preparation of all buffer solutions.

\subsection{Apparatus}

A LK2005A model potentiostat from lan li ke in tianjin driven by an PHILIPS PC with software was used for electropolymerization and detection measurements. A three-electrode cell with a saturated $\mathrm{Ag} / \mathrm{AgCl}$ reference electrode and aplatinum foil counter electrode was used. Electronic analytical bal- 
ance is the model of FA2004A from the company of shanghai Precision \& Scientific instrument. A PH meter is from Shanghai Magnetic Technology Co.,Ltd. Ultrasonic cleaning instrument is from Ningbo Chi Biotechnology Co.,Ltd.

\subsection{Characteristics and Functions of Material and Technology}

Polypyrrole has been in-depth studyed because of its excellent conductivity and simple synthetic process. It has been found that various additives and doped or composite nanoparticles in the polypyrrole ,not only improve the electrical conductivity of polypyrrole,but also enhance its thermal stability and mechaniccal ductility. In this research,we use the method of electrochemical polymerization to form a polypyrrole film on the surface of carbon electrode[5,6].

As a unique nano-materials, Carbon nanotubes has large specific surface area, excellent electrical,chemical properties and biological affinity. we can modify the carbon nanotubes through replacing, addition and oxidation, whether at the surface, the end or the tube, for introducing functional groups and bioactive component. This can be used for enzyme immobilization materials, or be served as the base electrode modified materials. In one word, we made a new type of carbon nanotube modified enzyme sensor[7].

Electrostatic self-assembly as a new biomolecular immobilization method has been increasingly used for the preparation of various biological sensors. With the principle of electrostatic self-assembly of supramolecular, it can make positive and negatively charged substances (nanoparticles, dyes, polyion, DNA, protein)attracting each other, and this method has more advantages considering biological activity, performance, stabil- ity, simple preparation,and preparation under mild condi- tions [8].

\subsection{Preparation of Enzyme Electrode[11,12]}

Polish the carbon electrode with $0.05 \mathrm{um} \mathrm{Al}_{2} \mathrm{O}_{3}$ suspension until it looks like a mirror, rinse clean,and then ultrasonic clean in distilled water and dilute sulfuric acid solution each with 10 minutes. Get out this processed eletrode,using the Three-electrode system to active, under $0.4--1.6 \mathrm{v}$ potential, with the speed of $50 \mathrm{mv} / \mathrm{s}$, scan by cyclic voltammetry in $0.5 \mathrm{~mol} / \mathrm{L}$ $\mathrm{H}_{2} \mathrm{SO}_{4}$ solution with 10 laps. After the activation electrode is removed, rinsed, and dried at room temperature. Then put this electrode into $0.1 \mathrm{~mol} / \mathrm{L}$ pyrrole solution scanning by cyclic voltammetry with 60 laps, at the speed of $100 \mathrm{mv} / \mathrm{s}$. Put this electrode modified with polyprrole into carbon nanotube solution for 12 hours, and then transfer it to Formaldehyde dehydrogenase solution for 24 hours under $4^{\circ} \mathrm{C}$, wash by stilled water, Finally drop nafion solution for capping,dry and put it in PBS solution under $4^{\circ} \mathrm{C}$ for using.

\subsection{The Preparation of the Substrate}

In this study, all substrates for detecting contant $0.1 \mathrm{~mol} / \mathrm{LPBS}$, $4.8 \mathrm{~mol} / \mathrm{LNAD}+, 1.0 \mathrm{~mol} / \mathrm{L}$ Glutathione, and with different concentration of formaldehyde.

\subsection{The Mathods of Detection}

The device of the three-electrode: the reference electrode is $\mathrm{Ag} / \mathrm{AgCl}$, the counter electrode is platinum electrode, the working electrode is carbon electrode modified by Formaldehyde dehydrogenase. Under the condition of experiment, we use cyclic voltammetry and square wave voltammetry to test with the PBS buffer solution at $\mathrm{PH}=7.0$, and the scanning potential is ranging from $0.4 \mathrm{v}$ to $-1.6 \mathrm{v}$ at the scanning speed of $50 \mathrm{mv} / \mathrm{s}$. The current response of NADH is record by the enzyme electrode. When the enzyme electrode is not used,stored it in PBS buffer solution under $4^{\circ} \mathrm{C}$ at the $\mathrm{PH}$ value of 7.0.

\section{Results and Discussion}

\subsection{The Preparation of Polypyrrole Film}

Cyclic voltammetry can make a very uniform film with eletroless polymerization. The cyclic voltammogram of polypyrrole modified carbon eletrodes is showed in Figure 1, the potential is between $0.4 \mathrm{v}$ and $-1.6 \mathrm{v}$, scanning speed is $100 \mathrm{mv} / \mathrm{s}$, the concentration of pyrrole is $0.1 \mathrm{~mol} / \mathrm{L}$ with 60 laps. According to this picture, there are two obvious reduction peak in near $-0.42 \mathrm{v}$ and $-0.78 \mathrm{v}$, and also a oxidation peak in $-0.42 \mathrm{v}$, with the gradual increase of the aggregate number of laps. Reduction peaks and oxidation peaks in varying degrees are reduced, introducing the conductive properties declined with the polypyrrole increased,and last tend to stability,but the conductivity is also exist.

\subsection{The Determination of the Test Potential}

In order to find out the potential of $\mathrm{NADH}$ in reactions, we used square wave voltammetry to test in PBS solution and substrate with formaldehyde and other accessory factors. In Figure $\mathbf{2}$, there are two lines ,the line with one peak is the modified electrode in PBS solution,the other is in the substrate. Both of this lines have the same reduction peak in $-0.6 \mathrm{v}$, which is because of PBS. The second line has two other peaks:-1.2v and $-1.5 \mathrm{v}$, in substrates with different concentration formaldehyde. The peak of $-1.2 \mathrm{v}$ is changed, and the peak of $-1.2 \mathrm{v}$ is stable, so we can see the peak can show the speed of production of $\mathrm{NADH}$.The peak in $-1.5 \mathrm{v}$ is not known of its mechanism.

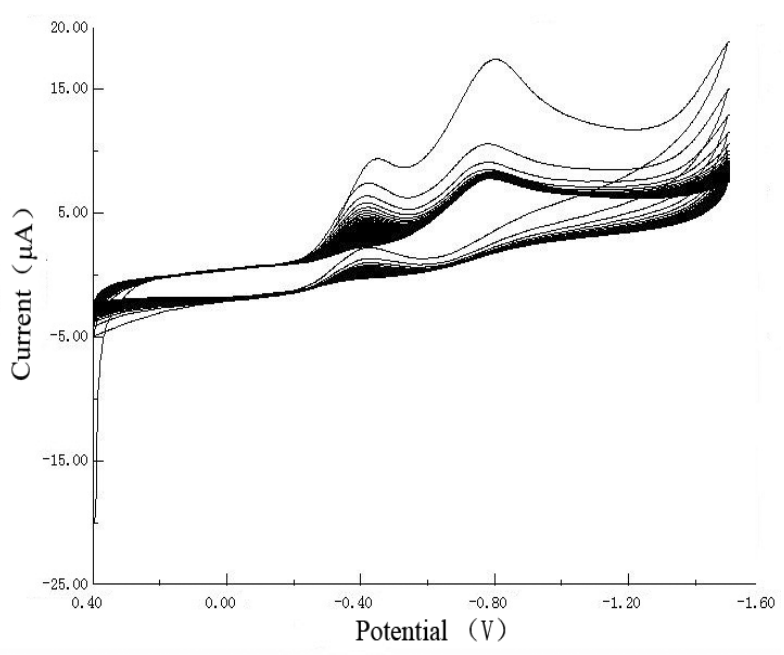

Figure 1. Electrochemical Polymerization of polypyrrole. 


\subsection{The Influence of Scanning Speed}

In the solution of substrate with $180 \mathrm{ug} / \mathrm{ml}$ formaldehyde, we used cyclic voltammetry to scan the potential between $0.4 \mathrm{v}$ and $-1.6 v$, with the speed from $50 \mathrm{mv} / \mathrm{s}-120 \mathrm{mv} / \mathrm{s}$. From the Figure 3 , we can see that, when the speed exchange, only the current peak increased, the potential peak is stable at nearly $-1.2 \mathrm{v}$, so we can say that the reaction is subject to the control of surface.

\subsection{The Electrochemical Response of the Sensor on Formaldehyde}

In this reseach, we detected different substrates with concentration: 50ug/ml, 20ug/ml, 15ug/ml, 10ug/ml, 6ug/ml, $1 \mathrm{ug} / \mathrm{ml}$, and responds were $22.967 \mathrm{uA}, 11.946 \mathrm{uA}, 10.542 \mathrm{uA}, 8.397 \mathrm{uA}$, 6.534uA, 4.628uA. From the Figure 4, the sensor to different concentration of formaldehyde shows a good liner relationship, and its standard curve equation is $\mathrm{Y}=0.3717 \mathrm{X}+4.5169$, with correlation coefficient of 0.9983 . The detection limit is $0.1 \mathrm{ug} / \mathrm{ml}$, and the detection ranges from $0.1 \mathrm{ug} / \mathrm{ml}$ to $360 \mathrm{ug} / \mathrm{ml}$.

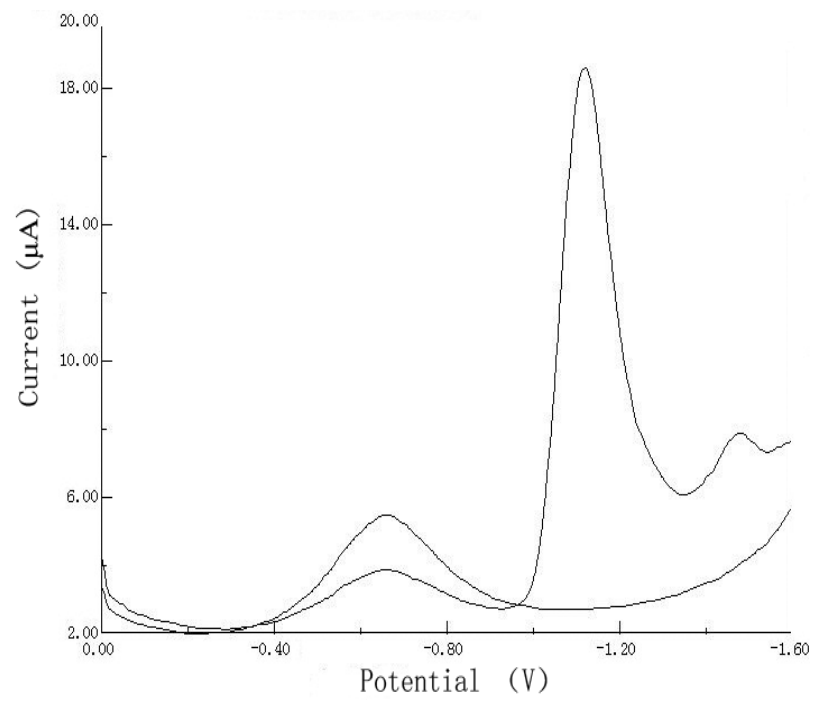

Figure 2. Testing in PBS solution and substrate of formaldehyde.

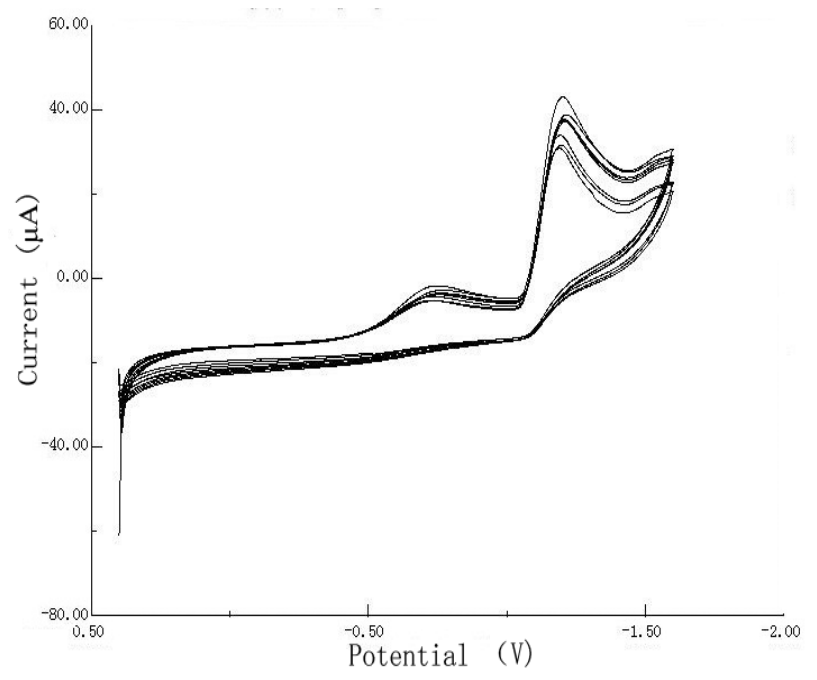

Figure 3. Different scanning speed for the electrode.

\subsection{The Influence of $\mathbf{P H}$}

Formaldehyde dehydrogenase catalyzed formaldehyde to formic acid ,which makes the solution PH value decline. However, the concentration of formaldehyde detected in the experimently is trace, and formic acid is a weak acid, so the $\mathrm{PH}$ value change is extremely small in reaction, it can be ignored. The only thing we must care about is the $\mathrm{PH}$ value of initial reaction substrate. In Figure 5, we used square wave voltammetry to detect response of enzyme electrode current in $180 \mathrm{ug} / \mathrm{ml}$ substrate, when other conditions excpt $\mathrm{PH}$ is unchanged. From the picture, we can see that the best response value of this eletrode is 7.5, indicating that formaldehyde dehydrogenase optimum $\mathrm{PH}$ value is 7.5 .

\subsection{Stability Test of Enzyme Electrode}

Put the enzyme eletrode at $4^{\circ} \mathrm{C}$ in PBS solution, using square wave voltammetry to detect substrate in the same concentration of formaldehyde at different times. From the pictrue of Figure 6, is the conclusion of the test at 1day, 10days,15days, 20days,

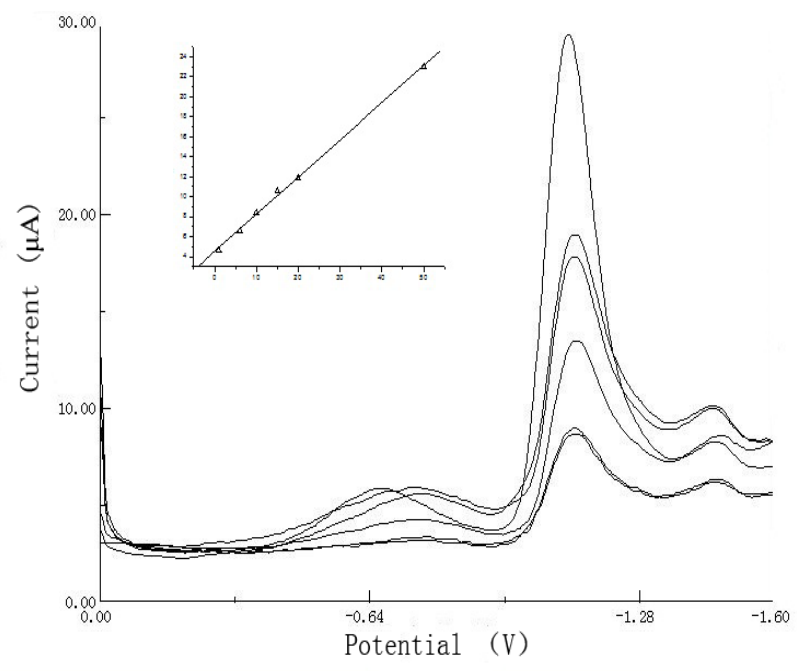

Figure 4. The liner response of different concentration range.

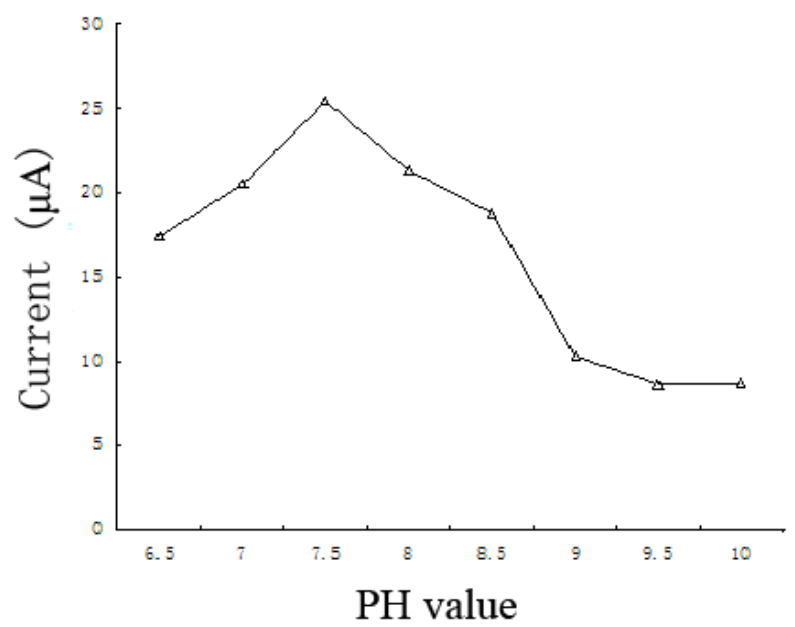

Figure 5. Influence of the Sensor by the PH value. 


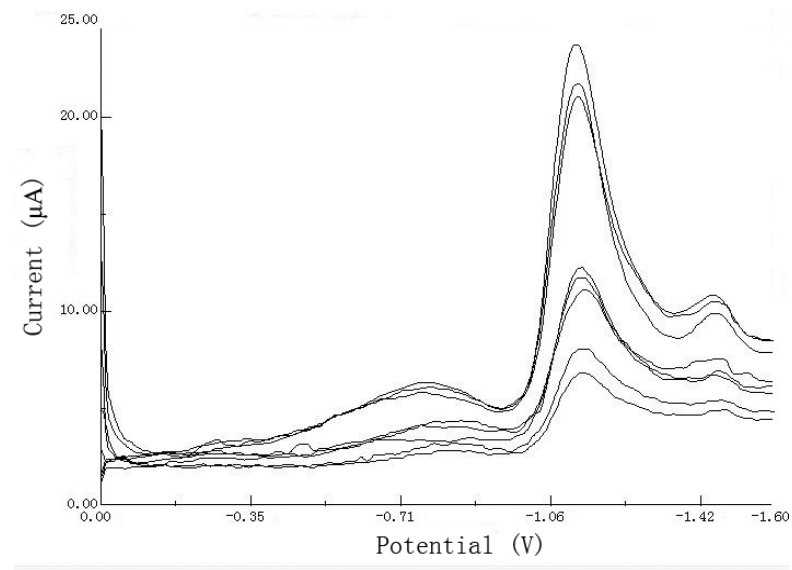

Figure 6. Testing at different times for stability.

25days, 30days, 40days, 45days, we can see that:the enzyme electrode at 1day, 10days, 15days is no obvious differece for testing the same substrate, but after 20days, the current is obvious reduced and has deviated from the standard curve, so the retention time of this electrode in $4{ }^{\circ} \mathrm{C}$ environment is 15 days.

\section{Conclusions}

Using polypyrrole as matrix, with the principle of electrostatic adsorption, we make the biosensor modified by enzyme for deteacting formaldehyde. In the biosensor,carbon nanotubes and formaldehyde dehydrogenase are immobilized on the surface of carbon eletrode, and nafion solution capped to prevent leakage of carbon nanotubes and enzyme. The sensor with formaldehyde dehydrogenase modified is simple and easy, with a good linear range, and also have practical value. However, there is some room for improvement. If we can fix $\mathrm{NAD}^{+}$and glutathione with formaldehyde dehydrogenase on the surface of electrode, we don't need to add them for test each time. The purity of enzyme solution is also need to improve, which may increase the response range of the substrate concentration.

\section{Acknowledgements}

The authors would like to thank the Ministry of Science and Technology of the People's Republic of China (2009GJA10047), Tianjin Municipal Science and Technology Commission (09ZCZDSFO4200), Tianjin Municipal Education Commission (SB20080035) and the Tianjin University of Science and Technology to support the work.

\section{REFERENCES}

[1] JIANG Zhongfa, YUAN Wenjun, ZHANG Benyan. Progress of study on the relationship between Indoor air formaldehyde Pollution and Leukemia[J] , Journal of Environment and Health, 2008,25(3): 276-278 (in Chinese).

[2] ZHAI, Jinxia, ZHANG Chuanmu, ZHANG Peng. Acute effects of formaldehyde in anatomy laboratory on student health[J]. Journal of Environment and Health, 2008,25(2): 141-143 (in
Chinese).

[3] Yang J, Zhang B, et a1. Cloning and Expression of Pseudomonas fluorescens 26-2 lipase gene in Pichla paaoris and characterizing for transosterification. Appl Biochem Biotechnol, 2008, 159(2): 355-365.

[4] Mitsubayashi K, NishioG, SawaiM, A bio-sniffer stick with FALDH (formaldehyde dehydrogenase)for convenient analysis of gaseous formaldehyde[J] . Sensors and ActuatorsB, 2008, 130( 1):32 37.

[5] Boukerma K, Piquemal J Y, Chehimi M M, et al. Synthesis and interfacial properties of montmorillonite/polypyrrolenanocomposites [J] . Polym. , 2006, 47:569-576.

[6] Ren X Z, Zhao Q, Liu J H, et al. Preparation of polypyrrole nanop-articles in reverse micelle anditsapplication to glucose biosensor $[\mathrm{J}]$. Journal of Nanoscience and Nanotechnology, doi:10. 1166/jnn. 2007(2):141.

[7] Wang S G,Zhang Q,Wang R L,et al.A Novel multi-walled carbonnanotube-based biosensor for glucose detection[J].Biochem BiophysRes Commun,2003,311(3):572 576.

[8] ZHANG Shu-ping, ZHAO Yan, MAJie1, LIUXiao-hui, WANGMeng-fei, LIUWei. Study on performance of layer-by-layer self-assembled electrodein detection of thiocholine[J].Modern Chemical Industry,2011,Jan,31(1):88 92

[9] William G.Gutheil, Elvin Kasimoglu, and Permila C.Nicholson. Induction of Glutathione-Dependent Formaldehyde Dehydrogenase Activity in Escherichia coli and Hemophilus influenza[J]. Biochemical and biophysical research communications 238, 693--696 (1997)

[10] William G.Gutheil,Barton Holmquist,and Bert L.Vallee. Purification, Characterization, and Partial Sequence of the Glutathione-Dependent Formaldehyde Dehydrogenase from Escherichia coli: A Class I11 Alcohol[J]. Biochemistry 1992, 31, 475-481.

[11] Shi yintao,Yuan ruo,Wang na, The biosensor based on polythionine Nano $\mathrm{Au}$ and horseradish peroxidase modified for detecting Hydrogen peroxide[J], Southwest China Normal University,2006,31,3.

[12] hou guoqing, The biosensor based on polyvinylpyrrolidone/Nano $\mathrm{Au}$ and horseradish peroxidase modified for detecting Hydrogen peroxide[J], Southwest China Normal University,2010,36,4.

[13] M. Ben Ali, M. Gonchar, G. Gayda, S. Paryzhak, M.A. Maaref, N. Jaffrezic-Renault, Y. Korpan . Formaldehyde-sensitive sensor based on recombinant formaldehydedehydrogenase using capacitance versus voltage measurements[J]. Biosensors and Bioelectronics 22 (2007) 2790-2795

[14] Kohji Mitsubayashi, Genki Nishio, Masayuki Sawai, Elito Kazawa . A biochemical sniffer-chip for convenient analysis of gaseousformaldehyde from timber materials[J]. Microchim Acta (2008) 160: 427-433.

[15] Lilach Bareket, Ada Rephaeli, Gili Berkovitch, Abraham Nudelman, Judith Rishpon. Carbon nanotubes based electrochemical biosensor for detection of formaldehyde released from a cancer cell line treated with formaldehyde-releasing anticancer prodrugs[J]. Bioelectrochemistry 77 (2010) 94-99.

[16] Yaroslav I. Korpan \& Olexandr O. Soldatkin \& Olga F. Sosovska \& Halyna M. Klepach . Formaldehyde-sensitive conductometric sensors based on commercial and recombinant formaldehyde dehydrogenase[J]. Microchim Acta (2010) 170:337-344. 